\title{
Expedient Construction of the Vibsanin E Core Without the Use of Protecting Groups
}

\author{
Ralf Heim, Stefan Wiedemann, Craig M. Williams, *and Paul V. Bernhardt \\ School of Molecular and Microbial Sciences, University of Queensland, St. Lucia, 4072, Queensland, Australia
}

\section{Experimental}

${ }^{1} \mathrm{H}$ and ${ }^{13} \mathrm{C}$ n.m.r spectra were recorded on Bruker AV400 $(400.13 \mathrm{MHz} ; 100.62 \mathrm{MHz})$, AV300 $(300.13 \mathrm{MHz} ; 75.47 \mathrm{MHz})$ and DRX500 $(500.13 \mathrm{MHz} ; 125.76 \mathrm{MHz})$ instruments in deuteriochloroform $\left(\mathrm{CDCl}_{3}\right)$. Coupling constants are given in $\mathrm{Hz}$ and chemical shifts are expressed as $\delta$ values in ppm. High and low resolution EI mass spectral data were obtained on a KRATOS MS 25 RFA. Microanalyses were performed by the University of Queensland Microanalytical Service.

\section{3-Methyl-3-(4-methylpent-3-enyl)cyclohexanone 8}

${ }^{1} \mathrm{H}$ NMR (400MHz, $\mathrm{CDCl}_{3}$ ) $\delta 0.91$ (s, 3H), 1.22-1.27 (m, 2H), 1.48-1.67 (m, $\left.2 \mathrm{H}\right), 1.56$ (bs, 3H), 1.62 (bd, J 1.2 Hz, 3H), 1.78-1.94 (m, 4H), 2.06-2.19 (m, 2H), 2.24 (bt, J 6.6, 2H), 5.02-5.07 (m, 1H). ${ }^{13} \mathrm{C}$ NMR $\left(100 \mathrm{MHz}, \mathrm{CDCl}_{3}\right) \delta 17.4,21.93,21.96,24.7,25.5,35.7,38.4,40.8,41.5,53.5$, 124.2, 131.3, 212.0. Mass spectrum m/z (EI) $194\left(\mathrm{M}^{+\bullet}, 7 \%\right), 151$ (8), 111 (100), 97 (5), 83 (6), 69 (23), 55 (18). Anal. Calcd. for $\mathrm{C}_{13} \mathrm{H}_{22} \mathrm{O}$ : C, 80.35; H, 11.41. Found: C, 80.25; H, 11.64.

\section{5-Methyl-5-(4-methylpent-3-enyl)-2-cyclohexenone 9}

${ }^{1} \mathrm{H}$ NMR $\left(\mathrm{CDCl}_{3}, 500 \mathrm{MHz}\right) \delta 1.00$ (s, 3H), 1.30-1.38 (m, 2H), 1.56 (bs, 3H), 1.64 (bd, J 1.1 Hz, $3 \mathrm{H}), 1.85-1.98(\mathrm{~m}, 2 \mathrm{H}), 2.13-2.17(\mathrm{~m}, 1 \mathrm{H}), 2.21-2.25(\mathrm{~m}, 1 \mathrm{H}), 2.27-2.33(\mathrm{~m}, 2 \mathrm{H})$, 5.01-5.06 (m, 1H), 5.99 (dt, J 2.0, $10.1 \mathrm{~Hz}, 1 \mathrm{H}), 6.81-6.85$ (m, 1H). ${ }^{13} \mathrm{C} \mathrm{NMR}\left(\mathrm{CDCl}_{3}, 125 \mathrm{MHz}\right) \delta 17.5,22.3$, 24.7, 25.6, 36.5, 38.1, 41.4, 50.1, 124.0, 129.0, 131.7, 148.2, 199.8. Mass spectrum m/z (EI) 192 $\left(\mathrm{M}^{+}, 12 \%\right), 149$ (10), 124 (25), 109 (100), 95 (5), 81 (13), 69 (18), 55 (10). Anal. Calcd. for $\mathrm{C}_{13} \mathrm{H}_{20} \mathrm{O}: \mathrm{M}^{+\bullet}$ 192.1514. Found: $\mathrm{M}^{+\bullet} 192.1507$.

\section{2-Hydroxymethyl-5-methyl-5-(4-methylpent-3-enyl)-2-cyclohexenone 10}

${ }^{1} \mathrm{H}$ NMR $\left(\mathrm{CDCl}_{3}, 500 \mathrm{MHz}\right) \delta 0.91$ (s, 3H), 1.22-1.29 (m, 2H), 1.48 (s, 3H), 1.56 (s, 3H), 1.79-1.90 (m, 2H), 2.10-2.30 (m, 4H), 2.94 (bs, OH), 4.14-4.15(m, 2H), 4.94-4.98 (m, 1H), 6.70-6.73 (m, 1H). ${ }^{13} \mathrm{C} \mathrm{NMR}\left(\mathrm{CDCl}_{3}, 125 \mathrm{MHz}\right) \delta 17.6,22.4,24.7,25.6,36.7,38.0,41.3,50.3,60.6,124.1$, 131.7, 137.5, 144.2, 200.4. Mass spectrum m/z (EI) $222\left(\mathrm{M}^{+\bullet}, 9 \%\right), 204$ (14), 189 (10), 164 (8), 161

\footnotetext{
* Author to whom correspondence should be addressed (c.williams3@uq.edu.au).
} 
(15), 139 (22), 121 (86), 109 (53), 97 (22), 83 (18), 69 (49), 55 (39), 41 (100). Anal. Calcd. for $\mathrm{C}_{14} \mathrm{H}_{22} \mathrm{O}_{2}$ : C, 75.63; H, 9.97. Found: C, 75.65; H, 10.27.

\section{1,7,7-Trimethyl-6-oxa-tricyclo[6.2.2.0 $\left.{ }^{4,9}\right]$ dodecan-3-one 11}

M.p. 61-61 ${ }^{\circ} \mathrm{C} .{ }^{1} \mathrm{H}$ NMR $\left(\mathrm{CDCl}_{3}, 500 \mathrm{MHz}\right) \delta 0.94$ (s, 3H), 1.04 (s, 3H), 1.20-1.32 (m, 3H), 1.24 (s, 3H), 1.49-1.59 (m, 3H), 1.76-1.80 (m, 1H), 1.93-1.95 (m, 1H), 2.07 (d, J 16.5 Hz, 1H), 2.29 (dd, J 16.5, $3 \mathrm{~Hz}, 1 \mathrm{H}), 2.66-2.70(\mathrm{~m}, 1 \mathrm{H}), 3.52(\mathrm{dd}, \mathrm{J} 3.6,11.9 \mathrm{~Hz}, 1 \mathrm{H}), 4.46$ (d, J $11.9 \mathrm{~Hz}, 1 \mathrm{H}) .{ }^{13} \mathrm{C} \mathrm{NMR}$ $\left(\mathrm{CDCl}_{3}, 125 \mathrm{MHz}\right) \delta$ 20.8, 22.8, 27.8, 31.0, 31.9, 33.9, 38.9, 41.46, 42.3, 46.4, 53.3, 58.6, 73.7, 210.4. Near IR (Nujol) $v\left(\mathrm{~cm}^{-1}\right)$ 1709, 1702, 1695. Mass spectrum m/z (EI) $222\left(\mathrm{M}^{+\bullet}, 14 \%\right), 207$ (18), 164 (10), 146 (3), 136 (4), 129 (6), 121 (6), 109 (8), 106 (10), 94 (100), 79 (14). Anal. Calcd. for $\mathrm{C}_{14} \mathrm{H}_{22} \mathrm{O}_{2}: \mathrm{M}^{+\bullet} 222.1614$. Found: $\mathrm{M}^{+\bullet} 222.1619$.

\section{2-Ethoxycarbonyl-1,7,7-trimethyl-6-oxa-tricyclo[6.2.2.0 $\left.0^{4,9}\right]$ dodecan-3-one 15}

${ }^{1} \mathrm{H}$ NMR $\left(\mathrm{CDCl}_{3}, 300 \mathrm{MHz}\right) \delta 0.99$ (s, 3H), 1.07 (s, 3H), 1.07-1.24 (m, 2H), 1.25 (s, 3H), 1.27 (t, J 7.1, 3H), 1.29-1.47 (m, 1H), 1.54-1.68 (m, 2H), 1.85 (dt, J 13.3, 3.2, 1H), 2.03-2.07 (m, 1H), 2.35$2.45(\mathrm{~m}, 1 \mathrm{H}), 2.67-2.75(\mathrm{~m}, 1 \mathrm{H}), 3.06(\mathrm{~s}, 1 \mathrm{H}), 3.58(\mathrm{dd}, \mathrm{J} 12.0,3.6,1 \mathrm{H}), 4.12-4.29(\mathrm{~m}, 2 \mathrm{H}), 4.44(\mathrm{~d}$, J 12.0, 1H). ${ }^{13} \mathrm{C} \mathrm{NMR}\left(\mathrm{CDCl}_{3}, 75 \mathrm{MHz}\right) \delta 14.2,20.6,22.5,27.5,29.1,31.8,34.6,37.5,42.3,43.2$, 46.2, 58.1, 60.6, 66.2, 73.5, 168.8, 204.5. Mass spectrum m/z (EI) $294\left(\mathrm{M}^{+\bullet}, 14 \%\right), 279$ (18), 276 (3), 266 (4), 249 (9), 236 (9), 233 (7), 221 (2), 205 (2), 190 (11), 181 (3), 175 (3), 162 (8), 143 (26), 134 (11), 115 (9), 109 (12), 94 (100), 79 (19). Anal. Calcd. for $\mathrm{C}_{17} \mathrm{H}_{26} \mathrm{O}_{4}: \mathrm{M}^{+\bullet}$ 294.1831. Found: $\mathrm{M}^{+\bullet}$ 294.1831.

\section{2-Ethoxycarbonyl-1,8,8-trimethyl-7-oxa-tricyclo[7.2.2.0 $\left.0^{5,10}\right]$ dodecan-3-one 20}

${ }^{1} \mathrm{H} \mathrm{NMR}\left(\mathrm{CDCl}_{3}, 300 \mathrm{MHz}\right) \delta 1.04(\mathrm{~s}, 3 \mathrm{H}), 1.09$ (s, 3H), 1.10-1.21 (m, 1H), 1.22-1.28 (m, 1H), $1.25(\mathrm{t}, \mathrm{J} 7.1,3 \mathrm{H}), 1.27(\mathrm{~s}, 3 \mathrm{H}), 1.31-1.47(\mathrm{~m}, 1 \mathrm{H}), 1.52-1.62(\mathrm{~m}, 1 \mathrm{H}), 1.73(\mathrm{dd}, \mathrm{J} 14.5,5.4,1 \mathrm{H})$, 1.95 (dt, J 14.5, 2.5, 1H), 2.16-2.25 (m, 2H), 2.54-2.62 (m, 1H), $2.63(\mathrm{~s}, 1 \mathrm{H}), 2.64(\mathrm{AB}, 1 \mathrm{H}), 3.05-$ $3.18(\mathrm{~m}, 1 \mathrm{H}), 3.62(\mathrm{dd}, \mathrm{J} 11.8,3.6,1 \mathrm{H}), 4.13$ (q, J 7.1, 2H), 4.31 (dd, J 10.7, 1.1, 1H). ${ }^{13} \mathrm{C}$ NMR $\left(\mathrm{CDCl}_{3}, 75 \mathrm{MHz}\right) \delta 14.2,21.9,23.8,27.4,31.2,33.0,33.7,34.2,42.8,44.3,46.4,51.3,51.6,60.4$, 61.7, 73.6, 174.0, 210.1. Mass spectrum m/z (EI) $308\left(\mathrm{M}^{+\bullet}, 4 \%\right), 293$ (14), 290 (4), 275 (1), 269 (1), 263 (5), 250 (68), 245 (1), 214 (1), 204 (19), 196 (6), 177 (10), 156 (90), 135 (10), 128 (18), 110 (23), 101 (94), 94 (100). Anal. Calcd. for $\mathrm{C}_{18} \mathrm{H}_{28} \mathrm{O}_{4}: \mathrm{M}^{+\bullet}$ 308.1988. Found: $\mathrm{M}^{+\bullet} 308.1989$. 